\title{
Software review: Optimising the allocation of cross-selling resource while managing the customer relationship
}

Received: 13th December, 2001

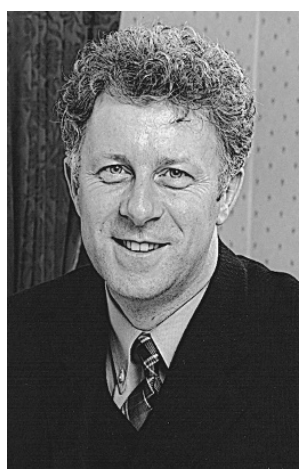

\section{Shaun Doyle}

is VP of Intelligent Marketing Solution at SAS. He is responsible for driving the application of SAS technologies in marketing. Prior to this role he was Chairman of Intrinsic, a campaign management software company. A co-founder of the company with Steve Treadwell, he was responsible for management of the business consultancy team and the strategic direction of the company. He has extensive experience in the design, development, implementation and exploitation of customer-focused database marketing systems in a number of business sectors. These include financial services, retail, leisure, charity and telecommunications. He has been involved in the design and building of over 50 marketing databases in blue chip organisations.

$\mathrm{He}$ is well known in the industry, regularly speaking and running specialist workshops at leading industry conferences. He has had a number of papers published in the UK and overseas on the application of database marketing.

\section{Abstract}

There has been significant interest expressed in the software review in Vol. 9, No. 2 of this journal on the use of optimisation techniques in marketing (Communication optimisation - The new mantra of database marketing. Fad or fact), so this paper explores further how optimisation could be used to support the allocation of cross-selling resource in an imaginary bank, Intel Financial Services Ltd. (IFS)

\section{INTRODUCTION}

With the wider availability of channels that can be used to send and receive communications with customers there has come a significant increase in the complexity of the communication decisioning process, ie deciding which customer gets what communication, when and by what channel. This has led to the development of a new range of optimisation technologies specifically developed for marketing. This

\section{Shaun Doyle}

SAS Software Ltd, Units 63-64 Shrivenham One Hundred Business Park, Shrivenham, Swindon, Wilts SL7 2EB.

Tel: +44 (0)1367 240003; Fax: +44 (0)1367 244604; e-mail: shaun.doyle@ suk.sas.com optimisation capability is concerned with allowing an organisation to:

- define global contact rules that manage the number of communications that a customer will receive within a defined period and the period between communications of a similar type

- determine the optimal product offering to be made to the customer at a given point in time. This opimisation process is typically being driven by behavioural models

- determine the most appropriate channel for the customer communication, taking into account customer preferences and channel and/or business constraints, eg budget

- ensure that the 'best' opportunities are sent to the channel, thereby maximising the productivity of the channel

- allocate opportunities to individual agents within a given channel

— distribute opportunities to primary 
and secondary channel assignments based on daily channel capacity and number of communication opportunities.

This paper explores the use of a well-known optimisation technique to optimise the allocation of cross-sell resource in a financial services organisation. The technique described here uses a simple linear programming routine to solve this common cross-selling business problem.

The solution optimally allocates resources based on the understanding achieved through the use of commonly used analytical customer relationship management (CRM) tools, and thus can be viewed as taking marketing analytics to the next level.

\section{BUSINESS SCENARIO}

A marketing analyst at Intel Financial Services, Ltd. (IFS) has used mining software (eg SAS Enterprise Miner or IBM Intelligent Miner) to uncover valuable information about IFS's customers and the types of products they own (or do not own). The marketing manager is interested in finding out how this information could be used to determine the optimal allocation of the limited cross-selling resources to market IFS's products. He is interested in achieving the highest possible returns (MROI) on the marketing resource invested. The following cross-sell optimisation solution will give the marketing manager the necessary guidance.

\section{BUSINESS PROBLEM}

Identifying cross-selling opportunities is an important goal of an analytical CRM solution. One way this can be done is with market basket analysis. Based on the principles of market basket analysis, the mining software produces rules data that show cross-selling opportunities. These rules are determined simply by counting the number of customers in the database that have the different combinations of products. The marketing analyst uses these counts to identify cross-selling opportunities. The rules, however, do not show which of these opportunities is best in meeting overall business goals, nor do they show how to distribute resources to achieve the business goal of maximising MROI.

The following is a list of products sold by IFS

- ATM (automatic teller machine card)

- MOTOR (motor loan)

- CCRD (credit card)

- DA (deposit account)

- CKCRD (cheque card)

- CKING (cheque account)

- HMEQLC (home equity loan)

- IRA (individual retirement account)

- MMDA (money market certificate)

- SVG (savings).

After running the mining software on the historical data, IFS's marketing director is presented with the following kinds of results: a rules dataset which contains association rules such as 'CKING to SVG \& CCRD' with a lift of 1.1. This is often interpreted to mean that the population that only has the cheque account (CKING) product has some potential, of strength 1.1 , for purchasing savings and credit cards. The rules dataset may contain hundreds if not thousands of such rules. While each rule is of value in identifying the potential of specific customer populations, the solution gives no suggestion as to whether this or any other rule should be used as a basis for expansion of just one of the target products, eg the savings market. 
Moreover, if a specific rule is used as a basis for allocating resources, it does not show how that decision will affect secondary target markets, for example the credit cards market, and whether exploiting this rule is a good overall use of limited resources.

Although this information is valuable, it leaves a fundamental business problem unanswered, ie how limited resources can best be allocated to exploit cross-selling opportunities that meet overall product sales goals. Addressing this business problem is typically not supported by campaign management technologies although it is likely to become a natural extension in the future.

\section{POTENTIAL SOLUTION OVERVIEW}

A simple approach based on the rules data set requires several assumptions. It is assumed that there are limited resources to spend on product marketing. For generality, this is called resource effort, and it is assumed that there is one unit available (this is 100 per cent selling effort). It is also assumed that there are target effort levels for each individual product. That is, a target percentage of effort to spend on selling each product is known.

Note that effort can be measured in many different kinds of units. It could be pounds, people or person hours. So, in the example, the marketing director at IFS has a budget of $\mathcal{2} 3 \mathrm{~m}$ to spend on product marketing. This $\mathcal{L}^{3} 3 \mathrm{~m}$ thus represents 100 per cent effort resource. In addition, the marketing director has specific product effort targets, for example the director wants to spend 500,000 marketing individual retirement accounts and $\mathcal{2}_{200,000}$ marketing savings accounts.

With these targets in mind, the director's business problem is the following; on which customer groups should selling efforts be focused in order to meet the targets for each product and, at the same time, maximise the return on effort investment (ie the $f 3 \mathrm{~m}$ )? This is called the objective, the director's objective.

The proposed cross-sell optimisation solution answers this by identifying the amount of effort to use on each customer group, for example spend $£ 100,000$ on marketing to the customer segment that only has savings and cheque accounts. The solution also meets the product sales targets while maximising the return on the $f 3 \mathrm{~m}$ marketing investment. Information about the size of the potential markets is incorporated implicitly in the objective through the lift. Since this is accounted for implicitly, the solution may recommend significant effort for customer groups simply because they have a large likelihood of success even though they do not represent a large market. To provide some control on this, a constraint is added that limits the average expected confidence weighted by effort to be less than a user-supplied quantity.

\section{SOLUTION METHODOLOGY}

The solution methodology is based on linear programming. A model that represents the business problem is built in the software. The solution of this model identifies an optimal distribution of effort across all rules that maximises the return on the investment. The model is supported by the data from the mining software and user-supplied parameters such as the product effort target levels, the anticipated returns from selling to different customer groups, and maximum acceptable average expected confidence. The solution is given as a vector of efforts to be applied to the different customer groups. The rules data set 
contains variables such as rule, expected confidence and lift. These are calculated from the raw data. Each record in the data set is a new rule that identifies a customer group or segment. The rules have left-hand side products and right-hand side products. The record gives information on the likelihood of selling the right-hand side products to customers who have the left-hand side products. Some of the measures for this include the lift and the expected confidence. The lift is the ratio of the probability of having the right-hand side products given that the customer has the left-hand side products over the probability that the customer has the right-hand side products. Thus a large value of lift indicates that the percentage of population with the left-hand side products is relatively small compared to the strength of the relationship between the right-hand side and left-hand side products.

\section{MODEL FORMULATION}

The solution approach is to build a linear programming model of the business problem. The model consists of an objective and a set of constraints. The objective drives the calculation of efforts and the constraints capture the business issues. The objective is to distribute 100 per cent selling effort across the multitude of cross-selling opportunities so that the weighted average of the product of lift and potential revenue is maximised. This average is weighted by the effort.

There are three types of constraints. One merely specifies that the total amount of effort is 1 . This defines the limited resources available for selling. Another restricts the average of expected confidence weighted by effort. This biases the effort towards customer populations that have greater growth potential. Finally, there is a set of constraints that requires a minimum amount of effort to be allocated to each product.

Let

$$
\begin{aligned}
J= & \text { set of products } j \\
I= & \text { set of rules } i \\
a_{i j}= & \left\{\begin{array}{l}
1 \text { if rule } i \text { has product } j \text { as a } \\
\text { result } \\
0 \text { otherwise }
\end{array}\right. \\
T_{j}= & \text { target effort for product } j \\
r_{i}= & \text { return from rule } i \text { if } 100 \% \text { effort } \\
& \text { is applied } \\
l_{i}= & \text { lift from rule } i \\
c_{i}= & \text { expected confidence of rule } i \\
C= & \text { maximum expected confidence } \\
& \text { for the weighted average effort } \\
& \text { allocation } \\
x_{i}= & \text { effort to apply to rule } i
\end{aligned}
$$

All of these quantities are known input parameters except for the effort $x_{\mathrm{i}}$. This is the quantity that is to be calculated by the system and is called the decision variable.

Formal specification has the objective as

$$
\operatorname{Max} \sum_{i \in I} r_{i} l_{i} x_{i}
$$

and the constraints as:

$$
\begin{array}{ll}
\sum_{i \in I} a_{i j} x_{i} \geq T_{j} \quad \forall j \in J & \begin{array}{l}
\text { Target product } \\
\text { efforts } \\
\sum_{i \in I} x_{i}=1
\end{array} \\
\sum_{i \in I} c_{i} x_{i} \leq C & \begin{array}{l}
100 \% \text { effort } \\
\text { available }
\end{array} \\
\text { Confidence limit } \\
x_{i} \geq 0 \quad \forall i \in I & \begin{array}{l}
\text { Nonnegative } \\
\text { effort }
\end{array}
\end{array}
$$

\section{SAS ROUTINE}

To illustrate this approach SAS code that reads the rules data set and builds the appropriate representation of the linear program described above has been 
Table 1

\begin{tabular}{llll}
\hline Rule set description & Effort & Lift & e Confidence \\
\hline CKING TO SVC \& CD & 0.1 & 1.06 & 15.72 \\
SVG \& CKING TO IRA & 0.1 & 1.01 & 10.84 \\
CKCRD TO CKING \& CCRD & 0.4 & 3.33 & 14.85 \\
CKING \& CCRD TO CKCRD & 0.1 & 3.33 & 11.30 \\
CKING \& CD TO MMDA & 0.1 & 1.25 & 17.44 \\
ATM TO CKING \& AUTO & 0.1 & 1.33 & 8.52 \\
SVG \& CKING TO HMEQLC \& ATM & 0.1 & 1.32 & 8.53 \\
\hline
\end{tabular}

Table 2

\begin{tabular}{lll}
\hline Product code & Product name & Effort \\
\hline ATM & Automatic teller machine card & 0.1 \\
MOTOR & Motor loan & 0.1 \\
CCRD & Credit card & 0.4 \\
DA & Deposit account & 0.1 \\
CKCRD & Check card & 0.1 \\
CKING & Checking account & 0.5 \\
HMEQLC & Home equity loan & 0.1 \\
IRA & Individual retirement account & 0.1 \\
MMDA & Money market certificate & 0.1 \\
SVG & Savings & 0.1 \\
\hline
\end{tabular}

written. Of course, the additional model parameters must also be specified. To simplify further, it is assumed that the product targets, $T_{j}$, are identical for all the products and that the returns, $R_{i}$, are one for each rule in the data set.

\section{EXAMPLE SOLUTION}

The following example demonstrates the use of this approach. The routine is called with an expected confidence level of 25 and an effort target of 10 per cent for each of the ten products that appear on the right-hand side of at least one rule in the data set.

The routine solves the problem by finding the distribution of effort that meets the constraints discussed above and maximises the total lift weighted by effort (since the returns $r_{i}$ are all one).

Tale 1 shows the solution. The program has picked out those customer populations that should be marketed or sold to. It shows the amount of effort to be applied to each of these targeted populations. The program picked populations that tend to have larger lift as would be expected, because of the objective. Also, most of the populations have effort 10 per cent, except for 'CKCRD to CKING \& CCRD' which has effort 0.4. Most probably this group is selected because of its high lift and low expected confidence.

In general, the program picks populations that have small expected confidence because of the constraint limiting the weighted average expected confidence to 25 .

Table 2 shows how the effort applied to these targeted populations translates into effort applied to the products. Note that each product has at least 0.1 effort as is required by the business objectives. Since some of the customer segments target multiple products the accounting may result in total effort over products exceeding 100 per cent. This is merely a result of the accounting. 


\section{OTHER OPTIONS}

As mentioned several times above, this is one method of solving this business problem and can serve as a starting point for exploration. Alternatives include:

- a constraint that seeks to ensure even dispersion of effort throughout all products

- a constraint that ensures a respectable return on equity. Because an organisation may be more interested in maintaining a certain level of shareholder value by avoiding inadequately performing products, rather than maintaining a diverse market of products

- finally, the use of a performance measure other than the lift as a measure of potential of the customer population. (For example, it may be more representative to use the lift factor divided by the maximum lift over all products, as a relative measure of potential).

\section{CONCLUSION}

This paper has illustrated how the use of optimisation techniques can be used effectively to resolve complex marketing problems. This is just one example that would benefit from further evaluation. But it shows that advanced analytics can play an important role in increasing effectiveness of marketing activities with minimal investment in technology. Perhaps the biggest issue is not the technology, but the realisation of the potential of advanced analytics.

CShaun Doyle

\section{Acknowledgments}

The author wishes to thank Marc Cohen, Leonardo Auslender and Judith-Marie Parks of the SAS Institute Ltd, SAS Campus Drive, Cary, NC 27513; Tel: +1 9196778000 for their help in preparing this paper. 